REVIEW

\title{
Postprandial dysmetabolism and cardiovascular disease in type 2 diabetes
}

\author{
M E Tushuizen, M Diamant, R J Heine
}

Postgrad Med J 2005;81:1-6. doi: 10.1136/pgmj.2004.020511

The worldwide prevalence of type 2 diabetes mellitus has reached epidemic proportions. The so-called traditional risk factors cannot fully explain the excessive cardiovascular disease risk of type 2 diabetic patients. Numerous studies indicate that postprandial metabolic derangements, most notably hyperglycaemia and hypertriglyceridaemia, which are exaggerated and prolonged in type 2 diabetes, are important cardiovascular disease risk factors since they induce oxidative stress and endothelial dysfunctions. This review discusses the current evidence showing that postprandial dysmetabolism may indeed constitute an important cardiovascular disease risk factor as well as the mechanisms underlying this association. Finally, some possible therapeutic options and recommendations for future research are discussed.

See end of article for authors' affiliations (n)

Correspondence to: Dr M E Tushuizen, Department of Endocrinology/Diabetes Centre, VU University Medical Centre, PO Box 7057, $1007 \mathrm{MB}$ Amsterdam, The Netherlands; m.tushuizen@vumc.nl

Submitted

12 February 2004 Accepted 22 April 2004
$\mathrm{T}$ he worldwide prevalence of type 2 diabetes mellitus is growing rapidly, reaching epidemic proportions. ${ }^{1}$ One of the major reasons of the increased prevalence in developing countries is the adoption of the so-called western lifestyle - that is, a high intake of energy dense food and a low physical activity pattern. These lifestyle changes lead to one of the key abnormalities underlying type 2 diabetes mellitus-that is, insulin resistance. Insulin resistance is associated with central obesity, hyperinsulinaemia, polycystic ovary syndrome, hypertension, and dyslipidaemia. $^{23}$ Hyperglycaemia, the established diagnostic marker of diabetes mellitus, is the result of the second key feature, progressive pancreatic $\beta$-cell failure.

It is well recognised that type 2 diabetic patients have an excess risk of developing atherosclerosis, resulting in high cardiovascular disease morbidity and mortality. ${ }^{4}$ Therefore, with the rise of the prevalence of diabetes, it may be expected that the global burden of cardiovascular disease will also increase. Since the so-called traditional risk factors such as high cholesterol, hypertension, smoking, and low high density lipoprotein (HDL) cholesterol, cannot fully explain the excessive cardiovascular disease risk of type 2 diabetic patients, other risk factors need to be identified. ${ }^{5}$

Two important processes involved in the development of atherosclerosis, the underlying cause of cardiovascular disease, are inflammation and coagulation activation. ${ }^{6}$ Although the exact mechanisms underlying atherogenesis are still not completely understood, vascular endothelial dysfunction is generally believed to be the starting point. ${ }^{67}$ Under normal conditions, the multiple functional characteristics of the endothelium, including regulation of the vascular tone, thrombogenesis, vascular wall permeability, and cell growth, collectively protect the vascular system. ${ }^{7}$ Adequate production of nitric oxide has a pivotal role in the majority of these processes. Since endothelial functions cannot be measured directly, several indirect methods have been developed to estimate these functions. These include the assessment of plasma concentrations of endothelial-cell derived proteins, including von Willebrand's factor (vWF), vascular cell adhesion molecule-1 (VCAM-1) and intercellular adhesion molecule-1 (ICAM-1), ${ }^{7}$ as well as the more complicated measurement of nitric oxide-dependent vasodilatation or flowmediated dilatation (FMD) of the brachial artery by non-invasive ultrasound. ${ }^{8}$ Increased plasma concentrations of VWF, VCAM-1 and ICAM-1, and a decreased FMD have been associated with an increased risk of cardiovascular disease. ${ }^{78}$

Other cardiovascular disease risk factors which have been recognised recently include hyperhomocysteinaemia, raised plasma levels of $\mathrm{C}$-reactive protein, reflecting a low grade inflammatory state, fibrinogen, representing a hypercoagulable state, and oxidised low density lipoprotein (LDL), as an indicator of oxidative stress. ${ }^{9}{ }^{10}$ However, only recently, the excessive and prolonged metabolic disturbances occurring in the postprandial state in type 2 diabetic subjects have regained interest as potential cardiovascular disease risk factors. ${ }^{11}$

Already over 200 years ago, William Heberden made the first observation regarding a postprandial effect on the circulation of blood, ${ }^{12}$ and in the late 1970s of the last century, Zilversmit postulated that atherosclerosis is a postprandial phenomenon. ${ }^{13}$ Ever since, a large body of evidence has accumulated indicating a relation between postprandial dysmetabolism, especially hyperglycaemia and hypertriglyceridaemia, and the risk of cardiovascular disease. ${ }^{114-17}$

Abbreviations: $2 \mathrm{hPG}$, two hour glucose concentrations after a 75 g glucose load; DECODE, Diabetes Epidemiology: Collaborative analysis Of Diagnostic criteria in Europe; FMD, flow mediated dilatation; HDL, high density lipoprotein; ICAM-1, intercellular adhesion molecule-1; LDL, low density lipoprotein; PAI-1, plasmin activator inhibitor-1; TRL, triglyceride rich lipoprotein; VCAM-1, vascular cellular adhesion molecule-1; VLDL, very low density lipoprotein; vWF, von Willebrand's factor 
In this review we will briefly discuss the present evidence for postprandial dysmetabolism as a potential cardiovascular disease risk factor, with special emphasis on postprandial glucose and lipid dysmetabolism. To this purpose, the association of postprandial dysmetabolism with the presence of (indicators of) atherosclerotic vascular disease and the possible underlying mechanisms will be reviewed.

\section{POSTPRANDIAL HYPERGLYCAEMIA}

Various mechanisms keep the plasma glucose levels in healthy subjects between strict limits, even after a carbohydrate load. The insulin response after a meal regulates the glucose uptake from the blood into the peripheral tissues and inhibits gluconeogenesis and glycogenolysis in the liver. Dysfunction of $\beta$-cells (for example, loss of the normal insulin secretion pattern) and insulin resistance contribute to glucose intolerance and both can be found very early in the disease process, finally leading to type 2 diabetes. ${ }^{2}$

In the United Kingdom Prospective Diabetes Study, lowering of glycated haemoglobin mainly reduced long term microvascular complications of diabetes, whereas effects on macrovascular disease were less convincing. ${ }^{18}$ In apparent contrast, several epidemiological studies have shown an association between two hour glucose concentrations after a $75 \mathrm{~g}$ glucose load (2hPG) and the occurrence of cardiovascular disease in the general population. ${ }^{19}{ }^{20}$ A meta-analysis of 20 studies with more than 95000 people demonstrated a continuous relationship between postload glucose levels and cardiovascular disease risk extending into the non-diabetic range. ${ }^{19}$ The Diabetes Epidemiology: Collaborative analysis Of Diagnostic criteria in Europe (DECODE) study demonstrated that the 2hPG concentrations, even in subjects with normal fasting glucose, were associated with mortality, independent of fasting plasma glucose concentrations. ${ }^{20}$

Chronic hyperglycaemia has been associated with impaired endothelial function. ${ }^{21}$ Recent studies in healthy and type 2 diabetic subjects indicate that acute hyperglycaemia causes endothelial dysfunction as measured by FMD. ${ }^{22}$ Also, circulating ICAM-1 plasma levels significantly increased in both diabetic and normal subjects after an oral glucose tolerance test, suggesting endothelial cell activation. ${ }^{23}$

Ceriello and co-workers have shown that postprandial hyperglycaemia is accompanied by several alterations of the coagulation system. ${ }^{24}{ }^{25}$ An oral glucose load in both healthy and type 2 diabetes patients caused a shortening of the half life of fibrinogen and an increase in plasma fibrinopeptide A and the fragments of prothrombin and factor VII. In addition, acute, short term hyperglycaemia resulted in a transient hyper-reactivity of platelets to high shear stress, combined with a significant rise of plasma vWF in patients with type 2 diabetes. ${ }^{26}$ Taken together, these findings suggest that hyperglycaemia may induce a hypercoagulable state.

In healthy subjects and those with impaired glucose tolerance, consecutive pulses of intravenous glucose increased circulating cytokine concentrations (interleukin-6 and tumour necrosis factor- $\alpha$ ) to a greater extent than during similar blood glucose levels which were kept stable during a hyperglycaemic clamp. This effect was more pronounced in subjects with impaired glucose tolerance. ${ }^{27}$ The same investigators showed changes in interleukin-6 (but not tumour necrosis factor- $\alpha$ ) plasma concentrations in type 2 diabetic patients after a carbohydrate meal. ${ }^{28}$ Thus, blood glucose excursions may induce a proinflammatory response.

Several in vitro studies demonstrated cytotoxic effects of high glucose levels in various cell types..$^{29-31}$ Of interest is the demonstration by Risso and colleagues that intermittent high glucose levels induced more apoptosis than constant corresponding glucose levels in human umbilical vein endothelial cells. $^{30}$
Four main molecular mechanisms underlying the hyperglycaemia-induced vascular damage have recently been reviewed, ${ }^{31}$ all of which are the result of intracellular hyperglycaemia. These include increased polyol pathway influx; increased advanced glycation end-product formation; activation of protein kinase $\mathrm{C}$ isoforms; and increased hexosamine pathway flux. These seemingly different mechanisms are the result of a single process - that is, overproduction of superoxide by the mitochondrial electron transport chain. This hyperglycaemia-induced oxidative stress ultimately results in modification of intracellular proteins resulting in an altered function, DNA damage, activation of the transcription factor nuclear factor- $\kappa \mathrm{B}$, causing abnormal changes in gene expression, decreased production of nitric oxide, and increased expression of cytokines, growth factors and procoagulant and proinflammatory molecules. ${ }^{31}$

Taken together, postload or postprandial glucose levels are associated with enhanced risk of cardiovascular disease. However, most epidemiological studies addressing the contribution of postload glucose levels to cardiovascular disease risk, especially the early ones, did not take into account the earlier mentioned classical risk factors, such as dyslipidaemia. In studies investigating the relationship between postload glucose and cardiovascular disease risk, adjustment for blood pressure, lipids and smoking, resulted in considerable attenuation of this association. ${ }^{32}{ }^{33}$ These data indicate that postload glucose may not be an independent cardiovascular disease risk factor but rather a risk marker, suggestive of underlying other metabolic disturbances, such as insulin resistance and dyslipidaemia, that may have an even greater impact on cardiovascular disease risk.

In conclusion, evidence of postprandial hyperglycaemia as an independent risk factor is not convincing. Therefore, the observed association between postload glucose excursions with cardiovascular disease is at least partly explained by the presence of insulin resistance and related cardiovascular disease risk factors.

\section{POSTPRANDIAL HYPERTRIGLYCERAEMIA}

In the western diet, more than $40 \%$ of the energy intake is derived from fats. Figure 1 demonstrates a schematic representation of the metabolic pathways of dietary fats leading to triglyceride-rich lipoproteins (TRL) and endogenous TRL production..$^{34}$ In the insulin-resistant state the production of very low density lipoprotein (VLDL) by the liver is inappropriately high. Together with a reduced lipoprotein lipase activity this results in high triglyceride concentrations, especially in the postprandial state. The large amount of TRLs and their prolonged residence time in the circulation may lead to increased exchange of the core lipid cholesteryl ester for triglycerides between TRL and LDL and HDL particles mediated by cholesteryl ester transfer protein. This process enriches LDL and HDL with triglyceride, and these particles are subsequently more readily hydrolysed by hepatic lipase resulting in smaller, denser LDL particles and lower concentrations of HDL. These abnormalities may explain the characteristic diabetic dyslipidaemia, which is now recognised to be very atherogenic. ${ }^{34}$

Already in 1959 an association between plasma concentrations and incident coronary heart disease was reported. ${ }^{35}$ However, the known inverse association between triglycerides and HDL cholesterol makes it difficult to show an independent association between plasma triglyceride and atherosclerotic vascular disease. A recently performed metaanalysis including data of 57000 subjects from 17 studies demonstrated that fasting triglyceride concentrations were an independent risk factor for cardiovascular disease, also when adjusted for HDL cholesterol. ${ }^{36} \mathrm{~A} 1 \mathrm{mmol} / \mathrm{l}$ increase in 


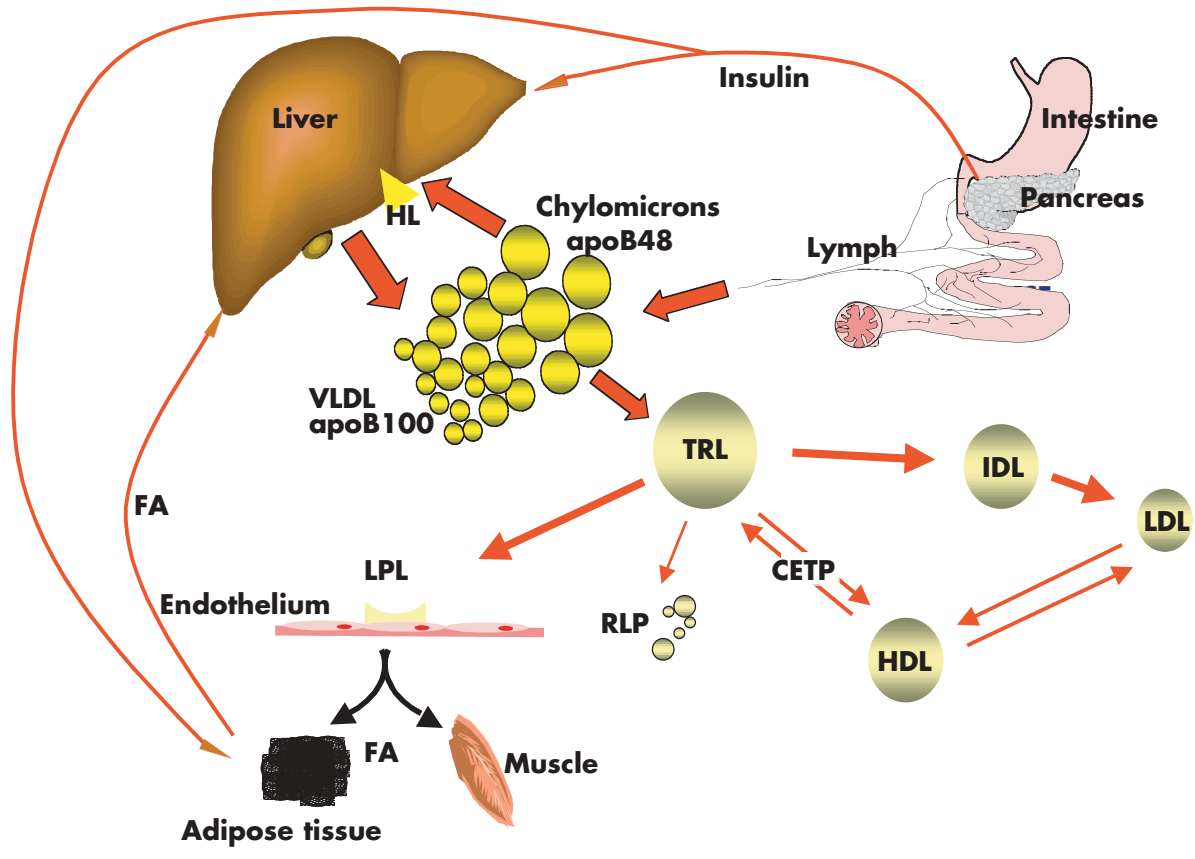

Figure 1 Lipid metabolism in vivo. Dietary fatty acids (FA) are absorbed from the gut and converted to triglycerides to be incorporated into chylomicrons in the intestinal epithelial cells. The triglyceride-rich apolipoprotein (apo) B48 containing chylomicrons enter the plasma via the intestinal lymph. Lipoprotein lipase (LPL) hydrolyses the triglyceride in chylomicrons to fatty acids, which are taken up by muscle cells for oxidation or adipocytes for storage. The remaining particles, the chylomicron remnants, are removed from the circulation by the liver through binding of their surface apoE to the low density lipoprotein (LDL) receptor or LDL receptor related protein. Very low density lipoprotein (VLDL) particles are triglyceride-rich apoB100 containing particles, synthesised by the liver. As with chylomicrons, VLDL triglycerides are hydrolysed by LPL. VLDL remnants or intermediate density lipoproteins (IDL) are taken up by liver receptors via apoE or converted to LDL. Chylomicrons, VLDL and their respective remnants (RLP, remnant lipoproteins) are termed triglyceride-rich lipoproteins (TRL). Under physiological conditions, insulin, which is raised in the postprandial state, suppresses lipolysis from adipose tissue and hepatic VLDL production, however, this insulin action is inappropriate in insulin resistance and type 2 diabetes, resulting in high TRL concentrations. The large amount of TRL and their prolonged residence time in the circulation increase the exchange of esterified cholesterol from high density lipoprotein (HDL) and LDL to TRL and of triglycerides to LDL and HDL particles, which is mediated by cholesterol-ester transfer protein (CETP). Triglyceride enrichment of LDL particles renders them better substrates for hepatic lipase (HL), which hydrolyses triglycerides from the core of LDL and turns them into smaller and denser particles. Small dense LDL are more atherogenic as they readily enter the subendothelial space and become oxidised. Triglyceride enriched HDL particles are smaller and are more rapidly catabolysed, which may explain the observed low plasma HDL in insulin resistance and type 2 diabetes. $^{34}$

plasma triglyceride was associated with a relative risk of 1.3 for men and 1.8 for women.

In general practice, serum lipid concentrations including triglycerides are measured in the morning after an overnight fast. However, the fasting value should be considered the nadir of the 24 hour triglyceride profile and could therefore be misleadingly low. In the past few years several clinical studies have suggested that high postprandial TRL may be related to coronary heart and/or carotid artery disease in nondiabetic and diabetic subjects. ${ }^{37-39}$ The Physician Health study, including 14916 men aged 40-84 years, with a follow up of seven years, showed that the non-fasting triglyceride concentrations strongly predicted incident myocardial infarctions, with a relative risk of 1.40 (95\% confidence interval 1.10 to 1.77 ) per $1.13 \mathrm{mmol} / \mathrm{l}$ increase. ${ }^{38}$ This study suggests that random or postprandial triglyceride concentrations are an important indicator of cardiovascular disease risk. Although fasting triglycerides are the most important determinant of postprandial triglycerides, ${ }^{40}$ it may be argued that in insulin resistant subjects with a delayed postprandial TRL clearance, non-fasting triglycerides should be used to approximate overall triglyceride exposure.

In male patients after a myocardial infarction, Karpe and co-workers found that the progression of coronary lesions over five years was related to the postprandial plasma levels of small chylomicron remnants (Sf 20-60 apolipoprotein B48). ${ }^{41}$ Adjustment for the possible confounding effect of HDL and dense LDL apolipoprotein B concentrations did not substantially alter the strength of this association. In line with these findings are coronary angiography data described by Mero et al, which suggest that especially small chylomicron remnants are implicated in the progression of coronary artery disease. ${ }^{16}$

In healthy and type 2 diabetic subjects, endothelial dysfunction, measured by ultrasound as FMD, has been associated with high triglyceride excursions. ${ }^{42}{ }^{43}$ Correlations between postprandial TRL, impaired FMD and oxidative stress markers have been demonstrated, suggesting that free radical production may be an underlying mechanism. ${ }^{42}{ }^{43}$ Supporting this hypothesis is the finding that in healthy subjects this effect could be attenuated by the antioxidant vitamin $C .{ }^{43}$

In vitro studies demonstrated increased adhesion molecule expression in endothelial cells after incubating with chylomicrons and VLDL. ${ }^{44}{ }^{45}$ Using rat arterial rings, Lundman et al showed impairment of endothelium-dependent relaxation following exposure to the triglyceride-containing fat emulsion Intralipid (Pharmacia-Upjohn, Uppsala, Sweden), however, exposure to VLDL did not affect vascular function. ${ }^{45}$

Postprandial coagulation activation by TRL was demonstrated by several investigators, however the underlying mechanism(s) are not fully understood ${ }^{46}{ }^{47}$ An elegant study performed by Silveira et al suggests an important role for the intrinsic coagulation pathway, based on in vivo activation of factor XI by triglyceride. ${ }^{46}$ Other prothrombotic changes occurring with an oral fat load are increased plasmin activator inhibitor-1 (PAI-1) activity and PAI-1 antigen. ${ }^{48}$ Postprandial lipaemia enhanced platelet P-selectin expression without affecting other markers of platelet activation. ${ }^{49}$ 


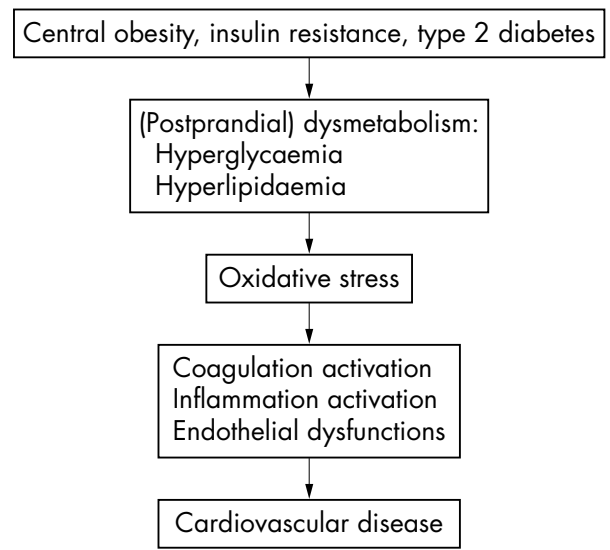

Figure 2 Insulin resistant states and type 2 diabetes are characterised by high circulating levels of atherogenic lipid particles due to an increased supply of fatty acids to the liver and defective hepatic clearance of lipoproteins. In the postprandial state, the lipid abnormalities are further exaggerated, with an additional adverse effect of meal induced hyperglycaemia. These postprandial metabolic derangements increase the production of reactive oxygen species causing oxidative stress and functional abnormalities of the vascular endothelium at several levels, including impairment of vasoreactivity, increased coagulation and inflammation activation, and increased vascular permeability. Collectively, postprandial dysmetabolism and the associated oxidative stress may link insulin resistance and type 2 diabetes to the disproportional incidence of cardiovascular disease in these high risk populations.

The effect of a high fat meal (50 g of fat) on cytokine concentrations, reflecting the inflammatory state, was studied in healthy and type 2 diabetic patients. ${ }^{28}$ In healthy subjects, significant correlations were found between postprandial triglyceride and tumour necrosis factor- $\alpha$ levels, whereas in diabetic patients also a positive correlation between postprandial plasma triglyceride and interleukin-6 concentrations was observed. Antioxidant supplementation lowered the rise of the cytokines, suggesting that the cytokine response to triglycerides was mediated by oxidative stress.

To summarise, historically, in diabetic patients, most emphasis was laid on hyperglycaemia, whereas recent evidence demonstrates the importance of dyslipidaemia, in particular hypertriglyceridaemia, as a cardiovascular disease risk factor. Although at present, epidemiological and long term intervention studies are largely lacking, in vivo data convincingly show an association between postprandial TRL and indicators of cardiovascular disease. Similar to postprandial hyperglycaemia, both in vivo and in vitro studies indicate that (postprandial) increases in triglycerides are proinflammatory, prothrombotic, and adversely affect several endothelial functions, by inducing oxidative stress (fig 2). Therefore, it is feasible that prolonged postprandial hypertriglyceridaemia leads to an atherogenic environment in vivo. However, as for postprandial hyperglycaemia the evidence for postprandial hypertriglyceridaemia as independent in cardiovascular disease is still scanty. More evidence, which can only be obtained from large prospective studies, is certainly required.

\section{THERAPEUTIC INTERVENTIONS}

Drugs that have been effective in reducing meal related glucose excursions are the $\alpha$-glucosidase inhibitors, the short acting insulin analogues, and the meglitinides. ${ }^{50-52}$

Recently, the STOP-NIDDM study showed a lower incidence of hypertension and myocardial infarction after treatment with the $\alpha$-glucosidase inhibitor acarbose. ${ }^{53}$ These findings should be interpreted with some caution as the study was not designed to assess the effect of acarbose on cardiovascular disease endpoints. The prospectively predefined endpoint was conversion to diabetes, however, in the final report 10 different cardiovascular disease endpoints were mentioned, including angina and peripheral vascular disease. In addition, it can not be excluded that the reduction in cardiovascular disease endpoints is the result of lowering triglycerides, since a triglyceride lowering effect of acarbose is described. ${ }^{54}$

Metformin is the only blood glucose-lowering drug that has been shown to lower diabetes related and cardiovascular disease endpoints in obese type 2 diabetic patients. Based on the proposed working mechanism, ${ }^{55}$ an effect on meal related glucose excursions can not be expected, but metformin was shown to reduce postprandial chylomicron concentrations. ${ }^{56}$

Correction of postprandial hyperglycaemia, for example with insulin secretion enhancers, will not only affect glucose levels but probably also the postprandial lipid responses. However, recently Vakkilainen et al demonstrated that nateglinide and glibenclamide increased postprandial insulin secretion and decreased postprandial glycaemia, but neither drug attenuated postprandial lipaemia in type 2 diabetic subjects with good glycaemic control. ${ }^{57}$

Intensive insulin treatment may improve diabetic dyslipidaemia to some extent, however, an entire correction of the atherogenic lipid profile, including postprandial hypertriglyceridaemia, may not be achieved.58

The lipid lowering drugs - that is, hydroxymethylglutaryl coenzyme A reductase inhibitors (statins), and peroxisome proliferator-activated receptor $\alpha$-agonists (fibrates), are established as most efficient agents that reduce cardiovascular disease morbidity and mortality in various high risk populations. ${ }^{5960}$ Statins inhibit cholesterol synthesis and upregulate the hepatic LDL receptor, whereas fibrates increase LPL activity and limit hepatic VLDL secretion. Based on their respective working mechanisms, the most benefit on postprandial lipidaemia may be expected from fibrates. Indeed, fibrate treatment reduced postprandial triglyceride levels by $30 \%-50 \% .{ }^{61}$ The proposed beneficial effect of fibrates on postprandial endothelial function measured by FMD, however, is disappointing. ${ }^{61}{ }^{62}$ Statins tend to induce a modest lowering of both fasting and postprandial triglycerides. ${ }^{61}{ }^{63}$ However, statin treatment showed a marked beneficial effect on postprandial induced oxidative stress and endothelial function. ${ }^{63}$ Independently of their cholesterol-lowering action, statins seem to have anti-inflammatory and vasculoprotective effects ("pleiotropic" effects).$^{64}$

We conclude that the high cardiovascular disease morbidity and mortality associated with type 2 diabetes is at least partly due to a prolonged and exaggerated postprandial state in these patients. To date, however, controlled randomised intervention studies showing that postprandial both glucose and triglyceride lowering results in amelioration of clinically relevant endpoints are lacking.

These conclusions should in no way distract from the therapeutic aim to achieve target glycated haemoglobin and lipid values in patients with type 2 diabetes.

\section{RECOMMENDATIONS FOR FUTURE RESEARCH AND TESTING POSTLOAD DYSMETABOLISM}

As discussed above, although the beneficial effect of therapy targeting postprandial dysmetabolism still needs to be established, studies assessing the true atherogenic exposure of the vascular system in high risk patients should abandon the classical glucose centred view and use physiological tests combining glucose and lipid loads. Most studies mentioned earlier demonstrated the effects of postprandial dysmetabolism on a single and rather artificial challenge, like a liquid $75 \mathrm{~g}$ glucose or liquid fat load. In daily life, most meals 


\section{Key references}

- Teno S, Uto Y, Nagashima $H$, et al. Association of postprandial hypertriglyceridemia and carotid intimamedia thickness in patients with type 2 diabetes. Diabetes Care 2000;23:1401-6.

- Ceriello A, Taboga C, Tonutti L, et al. Evidence for an independent and cumulative effect of postprandial hypertriglyceridemia and hyperglycemia on endothelial dysfunction and oxidative stress generation. Circulation 2002;106:1211-8.

- Heine RJ, Dekker JM. Beyond postprandial hyperglycaemia: metabolic factors associated with cardiovascular disease. Diabetologia 2002;45:461-75.

- Nappo F, Esposito K, Cioffi M, et al. Postprandial endothelial function in healthy subjects and in type 2 diabetic patients: role of fat and carbohydrate meals. $J$ Am Coll Cardiol 2002;39:1145-50.

- Taskinen MR. Diabetic dyslipidemia: from basic research to clinical practice. Diabetologia 2003;46: 733-49.

consumed are mixed and of solid consistence. Ceriello and co-workers showed a cumulative adverse effect of postprandial hypertriglyceridemia and hyperglycaemia on endothelial function..$^{63}$ The effect of a single component challenge possibly underestimates the real life postprandial dysmetabolic state and we therefore recommend the use of standardised mixed meal containing of at least $75 \mathrm{~g}$ of carbohydrates and $50 \mathrm{~g}$ of fat in future postprandial (intervention) studies.

\section{ACKNOWLEDGEMENTS}

MET is supported by a grant from the Dutch Diabetes Foundation (grant no 2000.00.025).

\section{QUESTIONS (TRUE/FALSE; ANSWERS AT END OF REFERENCES)}

1. In the next 30 years, the highest incidence of type 2 diabetes may be expected in the developed countries.

2. Hyperglycaemia is the result of insulin resistance.

3. The so-called traditional risk factors cannot fully explain the excessive cardiovascular disease risk in type 2 diabetic patients.

4. Endothelial functions can be measured directly in humans using ultrasound techniques.

5. Evidence suggests that high postload or postprandial glucose levels constitute an independent risk factor for the development of cardiovascular disease.

6. In the insulin resistant state, high VLDL concentrations are the result of an increased flux of chylomicrons from the gut due to a reduced lipoprotein lipase activity.

7. The fasting triglyceride value should be considered the nadir of the 24 hour triglyceride profile and could therefore be misleadingly low.

8. Statins have been shown to reduce postprandial triglyceride levels more adequately than fibrates and have a beneficial effect on postprandial induced oxidative stress.

9. The effect of a single nutrient component challenge possibly underestimates the impact of real life postprandial state.

\section{Authors' affiliations}

M E Tushuizen, M Diamant, R J Heine, Department of Endocrinology/ Diabetes Centre, VU University Medical Centre, Amsterdam, The Netherlands

\section{REFERENCES}

1 King H, Aubert RE, Herman WH. Global burden of diabetes, 1995-2025: prevalence, numerical estimates, and projections. Diabetes Care 1998;21:1414-31

2 Defronzo RA. Pathogenesis of type 2 (non-insulin-dependent) diabetes mellitus: a balanced overview. Diabetologia 1992;35:389-97.

3 Dunaif A. Insulin resistance and the polycystic ovarian syndrome: mechanisms and implications for pathogenesis. Endocr Rev 1997;18:774-800.

4 Haffner SM, Lehto S, Ronnemaa T, et al. Mortality from coronary heart disease in subjects with type 2 diabetes and in nondiabetic subjects with and without prior myocardial infarction. N Engl J Med 1998;339:229-34.

5 Stamler J, Vaccaro O, Neaton JD, et al. Diabetes, other risk factors, and 12-yr cardiovascular mortality for men screened in the Multiple Risk Factor Intervention Trial. Diabetes Care 1993;16:434-44.

6 Lusis A. Atherosclerosis. Nature 2000:407:233-41.

7 Widlansky ME, Gokce N, Keaney JF Jr, et al. The clinical implications of endothelial dysfunction. J Am Coll Cardiol 2003;42:1149-60.

8 Corretti MC, Anderson TJ, Benjamin EJ, et al. Guidelines for the ultrasound assessment of endothelial-dependent flow- mediated vasodilation of the brachial artery: a report of the International Brachial Artery Reactivity Task Force. J Am Coll Cardiol 2002;39:257-65.

9 Hackam DG, Anand SS. Emerging risk factors for atherosclerotic vascular disease: a critical review of the evidence. JAMA 2003;290:932-40.

10 Nishi K, Itabe $\mathrm{H}$, Uno $M$, et al. Oxidized LDL in carotid plaques and plasma associates with plaque instability. Arterioscler Thromb Vasc Biol 2002;22:1649-54

11 Ceriello $\mathbf{A}$. The post-prandial state and cardiovascular disease: relevance to diabetes mellitus. Diabetes Metab Res Rev 2000;16:125-32.

12 Heberden W. Some account of a disorder of the breast. Med Trans 1772;2:59-67.

13 Zilversmit DB. Atherogenesis: a postprandial phenomenon. Circulation 1979;60:473-85.

14 Haffner SM. The importance of hyperglycemia in the nonfasting state to the development of cardiovascular disease. Endocr Rev 1998;19:583-92.

15 Heine RJ, Dekker JM. Beyond postprandial hyperglycaemia: metabolic factors associated with cardiovascular disease. Diabetologia 2002;45:461-75.

16 Mero N, Malmstrom R, Steiner G, et al. Postprandial metabolism of apolipoprotein B-48- and B-100-containing particles in type 2 diabetes mellitus: relations to angiographically verified severity of coronary artery disease. Atherosclerosis 2000;150:167-77.

17 Karpe F. Postprandial lipoprotein metabolism and atherosclerosis. J Intern Med 1999;246:341-55.

18 UK Prospective Diabetes Study (UKPDS) Group. Intensive blood-glucose control with sulphonylureas or insulin compared with conventional treatment and risk of complications in patients with type 2 diabetes (UKPDS 33). Lancet 1998;352:837-53

19 Coutinho M, Gerstein HC, Wang Y, et al. The relationship between glucose and incident cardiovascular events. A metaregression analysis of published data from 20 studies of 95,783 individuals followed for 12.4 years. Diabetes Care 1999;22:233-40.

20 DECODE study group. Glucose tolerance and mortality: comparison of WHO and American Diabetes Association diagnostic criteria. European Diabetes Epidemiology Group, Diabetes Epidemiology: Collaborative analysis Of Diagnostic criteria in Europe. Lancet 1999-354:617-21

21 Thalhammer C, Balzuweit B, Busjahn A, et al. Endothelial cell dysfunction and arterial wall hypertrophy are associated with disturbed carbohydrate metabolism in patients at risk for cardiovascular disease. Arterioscler Thromb Vasc Biol 1999; 19:1173-9.

22 Kawano H, Motoyama T, Hirashima O, et al. Hyperglycemia rapidly suppresses flow-mediated endothelium-dependent vasodilation of brachial artery. J Am Coll Cardiol 1999;34:146-54.

23 Ceriello A, Falleti E, Motz E, et al. Hyperglycemia-induced circulating ICAM-1 increase in diabetes mellitus: the possible role of oxidative stress. Horm Metab Res 1998;30:146-9.

24 Ceriello A. Coagulation activation in diabetes mellitus: the role of hyperglycaemia and therapeutic prospects. Diabetologia 1993;36:1119-25.

25 Ceriello A, Giacomello R, Stel G, et al. Hyperglycemia-induced thrombin formation in diabetes. The possible role of oxidative stress. Diabetes 1995;44:924-8.

26 Gresele P, Guglielmini G, De Angelis M, et al. Acute, short-term hyperglycemia enhances shear stress-induced platelet activation in patients with type II diabetes mellitus. J Am Coll Cardiol 2003;41:1013-20.

27 Esposito K, Nappo F, Marfella R, et al. Inflammatory cytokine concentrations are acutely increased by hyperglycemia in humans. Circulation 2002; 106:2067-72

28 Nappo F, Esposito K, Cioffi M, et al. Postprandial endothelial function in healthy subjects and in type 2 diabetic patients: role of fat and carbohydrate meals. J Am Coll Cardiol 2002;39:1145-50.

29 Shizukuda Y, Reyland ME, Buttrick PM. Protein kinase C-delta modulates apoptosis induced by hyperglycemia in adult ventricular myocytes. Am J Physiol Heart Circ Physiol 2002;282:H1625-34.

30 Risso A, Mercuri F, Quagliaro L, et al. Intermittent high glucose enhances apoptosis in human umbilical vein endothelial cells in culture. Am J Physiol Endocrinol Metab 2001;281:E924-30.

31 Brownlee $M$. Biochemistry and molecular cell biology of diabetic complications. Nature 2001;414:813-20.

32 Bos G, Dekker JM, Niipels G, et al. A combination of high concentrations of serum triglyceride and non-high-density-lipoprotein-cholesterol is a risk factor for cardiovascular disease in subjects with abnormal glucose metabolismThe Hoorn Study. Diabetologia 2003;46:910-6. 
33 Meigs JB, Nathan DM, D'Agostino RB Sr, et al. Fasting and postchallenge glycemia and cardiovascular disease risk: the Framingham Offspring Study. Diabetes Care 2002;25:1845-50.

34 Taskinen MR. Diabetic dyslipidemia: from basic research to clinical practice. Diabetologia 2003;46:733-49.

35 Albrink MJ, Man EB. Serum triglycerides in coronary artery disease. Arch Intern Med 1959; 103:4-8.

36 Hokanson JE, Austin MA. Plasma triglyceride level is a risk factor for cardiovascular disease independent of high-density lipoprotein cholesterol level: a meta-analysis of population-based prospective studies. J Cardiovasc Risk 1996;3:213-9.

37 Groot PH, Van Stiphout WA, Krauss XH, et al. Postprandial lipoprotein metabolism in normolipidemic men with and without coronary artery disease. Arterioscler Thromb 1991;11:653-62.

38 Stampfer MJ, Krauss RM, Ma J, et al. A prospective study of triglyceride level, low-density lipoprotein particle diameter, and risk of myocardial infarction. JAMA 1996;276:882-8.

39 Teno S, Uto $\mathrm{Y}$, Nagashima $\mathrm{H}$, et al. Association of postprandial hypertriglyceridemia and carotid intima-media thickness in patients with type 2 diabetes. Diabetes Care 2000;23:1401-6.

40 Lewis GF, O'Meara NM, Soltys PA, et al. Fasting hypertriglyceridemia in noninsulin-dependent diabetes mellitus is an important predictor of postprandial lipid and lipoprotein abnormalities. J Clin Endocrinol Metab 1991;72:934-44.

41 Karpe F, Steiner G, Uffelman K, et al. Postprandial lipoproteins and progression of coronary atherosclerosis. Atherosclerosis 1994;106:83-97.

42 Anderson RA, Evans ML, Ellis GR, et al. The relationships between postprandial lipaemia, endothelial function and oxidative stress in healthy individuals and patients with type 2 diabetes. Atherosclerosis 2001;154:475-83.

43 Plotnick GD, Correti MC, Vogel RA. Effect of antioxidant vitamins on the transient impairment of endothelium-dependent brachial artery vasoactivity following a single high-fat meal. JAMA 1997;278:1682-6.

44 Moers A, Fenselau S, Schrezenmeir J. Chylomicrons induce E-selectin and VCAM-1 expression in endothelial cells. Exp Clin Endocrinol Diab 1997; 105(suppl 2):35-7.

45 Lundman P, Tornvall P, Nilsson L, et al. A triglyceride-rich fat emulsion and free fatty acids but not very low densitiy lipoproteins impair endotheliumdependent vasorelaxation. Atherosclerosis 2001;159:35-41.

46 Silveira A, Karpe $F$, Johnsson $H$, et al. In vivo demonstration in humans that large postprandial triglyceride-rich lipoproteins activate coagulation factor VII through the intrinsic coagulation pathway. Arterioscler Thromb Vasc Biol 1996;16:1333-9.

47 Silva KR, Kelly CN, Jones AE, et al. Chylomicron particle size and number, factor VIl activation and dietary monounsaturated fatty acids. Atherosclerosis 2003; 166:73-84.

48 Byrne CD, Wareham NJ, Martensz ND, et al. Increased PAl activity and PAI-1 occuring with an oral fat load: associations with PAI-1 genotype and plasma active TGF- $\beta$ levels. Atherosclerosis 1998; 140:45-53

49 Broijersen A, Karpe F, Hamsten A, et al. Alimentary lipemia enhances the membrane expression of platelet P-selectin without affecting other markers of platelet activation. Atherosclerosis 1998;137:107-13.

50 Kalbag JB, Walter YH, Nedelman JR, et al. Mealtime glucose regulation with nateglinide in healthy volunteers: comparison with repaglinide and placebo. Diabetes Care 2001;24:73-7.

51 Heinemann L, Klappoth W, Rave K, et al. Intra-individual variability of the metabolic effect of inhaled insulin together with an absorption enhancer. Diabetes Care 2000;23:1343-7.

52 Thompson RG, Pearson L, Schoenfeld SL, et al. Pramlintide, a synthetic analog of human amylin, improves the metabolic profile of patients with type 2 diabetes using insulin. The Pramlintide in Type 2 Diabetes Group. Diabetes Care 1998;21:987-93.

53 Chiasson JL, Josse RG, Gomis R, et al; STOP-NIDDM Trial Research Group. Acarbose treatment and the risk of cardiovascular disease and hypertension in patients with impaired glucose tolerance: the STOP-NIDDM trial. JAMA 2003;290:486-94.

54 Baron AD, Eckel RH, Schmeiser L, et al. The effect of short-term alphaglucosidase inhibition on carbohydrate and lipid metabolism in type II (noninsulin-dependent) diabetics. Metabolism 1987;36:409-15.

55 Kirpichnikov D, McFarlane SI, Sowers JR. Metformin: an update. Ann Intern Med 2002; 137:25-33.

56 Jeppesen J, Zhou MY, Chen YD, et al. Effect of metformin on postprandial lipemia in patients with fairly to poorly controlled NIDDM. Diabetes Care 1994; 17:1093-9.

57 Vakkilainen J, Mero N, Schweizer A, et al. Effects of nateglinide and glibenclamide on postprandial lipid and glucose metabolism in type 2 diabetes. Diabetes Metab Res Rev 2002;18:484-90.
58 Kudlacek S, Schernthaner G. The effect of insulin treatment on $\mathrm{HbAlc}$, body weight and lipids in type 2 diabetic patients with secondary-failure to sulfonylureas. A five year follow-up study. Horm Metab Res 1992;24:478-83. 59 Rubins HB, Robins SJ, Collins D, et al. Gemfibrozil for the secondary prevention of coronary heart disease in men with low levels of high-density ipoprotein cholesterol. Veterans Affairs High-Density Lipoprotein Cholesterol Intervention Trial Study Group. N Engl J Med 1999;341:410-8.

60 Collins R, Armitage J, Parish S, et al. MRC/BHF Heart Protection Study of cholesterol-lowering with simvastatin in 5963 people with diabetes: a randomised placebo-controlled trial. Lancet 2003;361:2005-16.

61 Karpe F. Postprandial lipemia-effect of lipid-lowering drugs. Atherosclerosis 2002; suppl:41-6.

62 Bae JH, Bassenge E, Lee HJ, et al. Impact of postprandial hypertriglyceridemia on vascular responses in patients with coronary artery disease: effects of ACE inhibitors and fibrates. Atherosclerosis 2001;158:165-71.

63 Ceriello A, Taboga C, Tonutti L, et al. Evidence for an independent and cumulative effect of postprandial hypertriglyceridemia and hyperglycemia on endothelial dysfunction and oxidative stress generation. Circulation 2002;106:1211-8.

64 Kinlay S, Selwyn AP. Effects of statins on inflammation in patients with acute and chronic coronary syndromes. Am J Cardiol 2003;91:9B-13.

\section{ANSWERS}

1. False. The excessive global increase in the occurrence of type 2 diabetes may be explained by the vast spreading of western lifestyle patterns, including consumption of energy dense foods and less physical activity, in parts of the world with the highest population densities-that is, the developing countries.

2. False. Hyperglycaemia is the result of progressive pancreatic $\beta$-cell failure.

3. True. Absolute risk of death by cardiovascular disease was much higher for diabetic than non-diabetic men of every age stratum, ethnic background, and risk factor leveloverall three times higher, with adjustment for age, race, income, serum cholesterol level, systolic blood pressure, and smoking. ${ }^{5}$

4. False. Endothelial function can only be estimatedindirectly-by provoking the endothelium to release nitric oxide resulting in vasodilatation that can be imaged and quantitated.

5. False. Postload glucose may not be an independent cardiovascular disease risk factor but rather a risk marker, suggestive of underlying metabolic disturbances that collectively impact on cardiovascular disease risk.

6. False. In the insulin resistant state inappropriate production of VLDL by the liver occurs, due to impaired suppression of VLDL triglyceride by insulin (hepatic insulin resistance) and an increased substrate (fatty acid) flux to the liver.

7. True. In clinical practice triglycerides are measured in the morning after an overnight fasting period. Triglyceride profiles of type 2 diabetic patients show that after breakfast their concentrations gradually increase after consecutive meals and the peak concentration is achieved between dinner and bedtime.

8. False. Based on their working mechanism, that is, increasing (postprandial) lipoprotein lipase activity, but also in practice, fibrates, rather than statins reduce postprandial triglycerides. Statins tend to induce a modest lowering of fasting and postprandial triglyceride levels.

9. True. A cumulative effect of postprandial hypertriglyceridaemia and hyperglycaemia on endothelial function has been demonstrated, possibly due to potentiation of oxidative stress mechanisms. 